\title{
Distrofia Simpático Reflexa no Pé e Tornozelo*
}

Imamura, M.*

Ignácio, L.A.*

Salomão, O.*

Chung, T. M.*

Teixeira, M. J.*

Imamura, $\mathrm{S}$. T.*

\section{RESUMO}

A etiologia da distrofia simpático reflexa (DSR) é desconhecida, porém a teoria mais aceita é a de uma disfunção do Sistema Nervoso Simpático. Os achados clínicos caracterizam-se por dor neuropática, distúrbios nasomotores e sudomotores e alterações tróficas da pele que aumentam em estágios mais avançados. O diagnóstico é feito através da história clínica, exame físico, radiografias, cintilografia óssea e termografia. $\mathrm{O}$ tratamento baseia-se em meios físicos, como hidroterapia e exercícios terapêuticos. Quando há necessidade são utilizados medicamentos que interferem no sistema modulador da dor como os antidepressivos tricíclicos e os neurolépticos.

O objetivo deste estudo é o de apresentar os resultados do tratamento de 13 pacientes com DSR que foram submetidos a meios físicos e terapia medicamentosa quando necessário.

\section{UNITERMOS}

Distrofia simpático reflexa. Analgesia. Dor em membros inferiores (extremidades).

\section{SUMMARY}

The etiology of the reflex sympathetic distrophy (RSD) is unknown but the most acceptable theory is that it is a sympathetic nervous system dysfunction. The clinical findings are characterized by neuropathic pain, vasomotor and sudomotor disturbances and trophic skin changes that are increased in the latter stages. Diagnoses is made by clinical history, physical examination, $\mathrm{x}$-ray, bone scan, thermography. The treatment is based on physical agents, such as hidrotheraphy and therapeutic exercises. When necessary medications which interferes in the pain modulating system, as tricyclic antidepressant and neuroleptics are used. The aim of this study is to show the results of the treatment of 13 patients with RSD that underwent to physical agents and drug therapy when necessary.

\section{KEY WORDS}

Reflex sympathetic distrophy. Analgesia. Lower limb pain.

\section{Introdução}

A distrofia simpático reflexa (DSR) é uma doença cuja etiologia ainda permanece obscura porém a hipótese mais aceita é a de que se trata de uma atividade anormal do sistema nervoso simpático. O quadro clínico é caracterizado por dor de origem neuropática, alterações vaso e sudomotoras, assim como alterações trófiicas que principalmente nas fases finais são muito acentuadas. O diagnóstico é feito pela história clínica, exame físico, raio-x, cintilografia óssea e estudo com placa termográfica. O tratamento é feito com medidas de medicina física, do tipo hidrotermoterapia 
e cinesioterapia; quando necessário são utilizados medicamentos para estimular o sistema supressor de dor do tipo neurolépticos e antidepressivos tricíclicos.

O objetivo deste trabalho é o de demonstrar os achados clínicos, etiologia e o resultado do tratamento de 13 doentes portadores de DSR no pé e tornozelo, que foram submetidos a medidas de Medicina Física e tratamento medicamentoso, quando necessário.

\section{Material e Método}

Foram acompanhados 13 doentes do grupo de pé do Instituto de Ortopedia e Traumatologia do Hospital das Clínicas da FMUSP, no período de setembro de 1992 a outubro de 1993, que apresentavam diagnóstico de DSR no pé e osteo-articular, verificou-se que a alteração inicial foi em ossos do pé em $9(69,2 \%)$ casos e em ossos da perna em 2 casos. Notou-se predominância do lado esquerdo que estava acometido em 9 casos sendo os $4(30,8 \%)$ restantes do lado direito. Não houve casos com comprometimento bilateral (Tabela 1). Quando os pacientes chegaram no nosso serviço, $2(15,4 \%)$ encontravam-se na fase I, $7(53,8 \%)$ na fase II e $3(23,0 \%)$ pacientes na fase III, sendo que em $1(7,7 \%)$ caso não houve relato.

O intervalo entre o início da sintomatologia e o início do tratamento foi de 1,5 a 10 meses (média = 5,2 meses). O tempo de acompanhamento variou de 1 a 10 meses (média= 3,6 meses).

Todos os pacientes foram submetidos a medidas de medicina física do tipo hidrotermoterapia e cinesioterapia, visando a analgesia e a recuperação da amplitude de

\section{TABELA 1 - DISTRIBUIÇÃO DE FREQÜÊNCIA SEGUNDO O LADO ACOMETIDO}

\begin{tabular}{ccc}
\hline Lado & Freqüência & Porcentagem (\%) \\
\hline Esquerdo & 9 & 69,2 \\
Direito & 4 & 30,8 \\
\hline
\end{tabular}

TABELA 2 - DISTRIBUIÇÃO DE FREQÜÊNCIA SEGUNDO A FASE DA DSR

\begin{tabular}{lcc}
\hline Fase & Freqüência & Porcentagem $(\%)$ \\
\hline I & 2 & 15,4 \\
II & 7 & 53,8 \\
III & 3 & 23,0 \\
sem relato & 1 & 7,7 \\
\hline
\end{tabular}

tornozelo. Sete pacientes eram do sexo feminino e cinco eram do sexo masculino. A idade variou de 24 a 73 anos, com média de 44,6 anos. O estudo da casuística está representado no Quadro 1.

O diagnóstico de DSR foi realizado através da história clínica, exame físico, estudo radiológico, cintilográfico e em placa termográfica.

A etiologia da DSR foi traumática em 9 casos $(69,2 \%)$, em $3(23,0 \%)$ casos ocorreu após artrodese tríplice para correção de deformidades estruturadas do pé em doentes portadores de paralisia infantil e em um caso após injeção intramuscular com lesão do nervo ciático.

Excetuando-se o caso por lesão nervosa, naqueles outros onde houve comprometimento movimento articular e do trofismo muscular. Destes doentes $3(23,0 \%)$ não responderam adequadamente ao tratamento acima e fizeram uso de medicação analgésica de ação central, do tipo antidepressivo tricíclico e em um paciente foi associado um neuroléptico fenotiazínico.

Nenhum doente foi submetido a acompanhamento psicológico. O bloqueio simpático não foi realizado em nenhum dos casos.

\section{Resultados}

Para avaliar os resultados foi considerado a melhora do quadro doloroso analisada segundo o 
QUADRO I

\begin{tabular}{|c|c|c|c|c|c|c|c|c|c|c|}
\hline $\begin{array}{l}\text { iniciais } \\
\text { (nome) }\end{array}$ & $\begin{array}{l}\text { número de } \\
\text { registro }\end{array}$ & I & $\mathbf{S}$ & etiologia & T1 & diagnóstico & $\begin{array}{l}\text { local } \\
\text { lesão }\end{array}$ & TX & $\mathrm{T} 2$ & $\%$ \\
\hline 1.IB & $4062791 \mathrm{I}$ & 73 & $\mathrm{~F}$ & queda altura & 2 & f. trimaleolar D & pé $\mathrm{D}$ & $\mathrm{C} / \mathrm{T}$ & 10 & 50 \\
\hline 2.FLO & $2859791 \mathrm{~F}$ & 49 & M & injeção IM & 10 & $\begin{array}{l}\text { lesão nervo } \\
\text { ciático }\end{array}$ & glú-teo & $\mathrm{C} / \mathrm{F}$ & 2 & 40 \\
\hline 3.CTQ & $4063960 \mathrm{~F}$ & 33 & M & entorse & 1,5 & lesão-ligamentar & pé $\mathrm{E}$ & $\begin{array}{l}\mathrm{C} / \mathrm{T} / \\
\mathrm{AD} / \mathrm{N}\end{array}$ & - & - \\
\hline 4.MGVM & $4063129 \mathrm{H}$ & 56 & $\mathrm{~F}$ & queda altura & 4 & f.l.e. trimaleolar & pé E & $\mathrm{C} / \mathrm{T}$ & 4 & 90 \\
\hline 5.ABSF & $4059352 \mathrm{G}$ & 24 & M & $\begin{array}{l}\text { sequela } \\
\text { PI MIE }\end{array}$ & 7 & $\begin{array}{l}\text { astrodese } \\
\text { tríplice }\end{array}$ & pé E & $\mathrm{C} / \mathrm{T}$ & 4 & 100 \\
\hline 6.MAFS & 4061317D & 53 & $\mathrm{~F}$ & queda altura & 5 & f. ossos perna E & perna $\mathrm{E}$ & $\mathrm{C} / \mathrm{T}$ & 6 & 70 \\
\hline 7.CFA & 4063761 & 43 & $\mathrm{~F}$ & atropelamento & 11 & f. e. tornozelo E & perna $E$ & $\mathrm{C} / \mathrm{T}$ & 6 & 40 \\
\hline 8.FVL & $5002852 \mathrm{~K}$ & 62 & M & entorse & 7 & $\begin{array}{l}\text { f. l. bimaleolar } \\
\text { tornezelo D }\end{array}$ & pé $D$ & $\mathrm{C} / \mathrm{T}$ & 1 & 100 \\
\hline 9. WVP & $4063799 \mathrm{G}$ & 29 & M & atropelamento & 6 & f. ossos perna $\mathrm{E}$ & perna $\mathrm{E}$ & $\begin{array}{l}\mathrm{C} / \mathrm{T} / \\
\mathrm{AD}\end{array}$ & 1 & 60 \\
\hline 10.EM & 2124956B & 29 & $\mathrm{~F}$ & queda de altura & 2 & $\begin{array}{l}\text { f. calcâneo E } \\
\text { astrodese tríplice }\end{array}$ & pé E & $\mathrm{C} / \mathrm{T} / \mathrm{F}$ & 2 & 60 \\
\hline 11.JDP & $4056258 \mathrm{I}$ & 35 & $\mathrm{~F}$ & sequela PI MIE & 4 & astrodese tríplice & pé E & $\mathrm{C} / \mathrm{T}$ & 2 & 60 \\
\hline 12.JE & $4064120 \mathrm{~A}$ & 51 & M & queda de altura & 6 & $\begin{array}{l}\text { f. tuberosidade } \\
\text { calcâneo D }\end{array}$ & pé $\mathrm{D}$ & $\mathrm{C} / \mathrm{T}$ & 2 & 40 \\
\hline 13.MFR & 4059929G & 33 & $\mathrm{~F}$ & sequela PI MID & 3 & astrodese tríplice & pé $\mathrm{D}$ & $\mathrm{C} / \mathrm{A}$ & 4 & 70 \\
\hline
\end{tabular}

abreviaturas usadas:

- I: idade. S: sexo.

- T1: intervalo de tempo entre o início dos sintomas e o início do tratamento (em meses).

- T2: duração do tratamento (em meses).

- TX: tratamento C: cinesioterapia T: turbilhão. F: corrente farádica. AD: antidepressivo tricíclico. N: neuroléptico.

- f.: fratura. l.: luxação. e.: exposta.

- \%: porcentagem de melhora.

- PI: paralisia infantil. IM: intramuscular. MID: membro inferior direito. MIE: membro inferior esquerdo.

- E: esquerdo. D: direito.
VAS (Escala Visual Analógica), onde é dada uma nota subjetiva, pelo próprio paciente, e que varia de $\mathrm{O}$ (nenhuma dor) a 10 (dor intensa). Além disso, foi feita uma avaliação médica em relação a melhora das alterações tróficas e da mobilidade articular.

Os resultados foram quantificados segundo a informação dos doentes de acordo com a porcentagem de melhora em relação à dor original.

Quando esta melhora, medida em percentagem, era de 80 a $100 \%$ consideramos um resultado ótimo; 50 a 79\% bom; 25 a 49\% regular; O a $24 \%$ mau.

Em 25\% dos casos (3 pacientes, dos quais nenhum fez uso de medicação) o resultado foi excelente, em 50\% (6 pacientes, destes 3 com medicamento) foi bom, em $25 \%$ (3 pacientes, dos quais nenhum fez uso de medicação) foi regular. Um paciente não completou o tratamento. álgico.

Nenhum paciente apresentou piora do quadro 


\section{Discussão}

A DSR é uma síndrome álgica incapacitante que impossibilita o indivíduo a exercer suas atividades de vida diária. A International Association for the Study of Pain (IASP) define a DSR como uma dor contínua em parte de uma extremidade, após trauma, que pode incluir fraturas, porém, sem o acometimento nervoso, associada a uma hiperatividade simpática ${ }^{(3)}$. Geralmente ocorre o acometimento da extremidade distal adjacente a área traumatizada.

Segundo Eulry et al. (1990)(1), ocorre uma maior incidência de DSR em pacientes jovens, do sexo masculino, de etiologia traumática. Na nossa casuística, apesar de a etiologia ser concordante com a literatura, houve predomínio no sexo feminino. Além da etiologia traumática, caracterizada por fratura dos ossos da perna e do pé, observamos a doença após a realização de artrodese tríplice.

As alterações distróficas da pele estavam presentes em $100 \%$ dos casos. A dor é um sintoma freqüente se não invariável na DSR. Ela esteve presente em $92,3 \%$ dos nossos casos desde o início do quadro. A dor inicia-se semanas após a lesão e é descrita como queimor, contínua, exacerbada pelo movimento, estimulação cutânea ou estresse emocional $^{(3)}$.

A DSR é dividida arbitrariamente em 3 fases $^{(2)}$ :

- Fase I: é o estágio agudo, que se inicia após a lesão, onde nota-se a presença de dor espontânea e hiperpatia; pele quente, seca e com hiperemia (inicialmente) e fria, cianótica e sudorética (tardiamente) com edema local e aumento do crescimento de unhas e pelos.

- Fase II: é o estágio distrófico. Inicia-se cerca de 3 a 6 meses após a lesão. Caracterizada por dor espontânea que pode se irradiar para regiões adjacentes; unhas quebradiças, rachadas com diminuição do crescimento de pelos; diminuição de amplitude de movimento articular, perda muscular, osteoporose; edema difuso.

- Fase III: é o estágio atrófico. Ocorre, geralmente, 6 meses após a lesão e é caracterizada por dor que pode ser menos intensa que nos estágios anteriores; diminuição da temperatura da pele, com cianose e palidez; alterações tróficas irreversíveis em pele e tecido celular subcutâneo; atrofia subcutânea; rigidez e contratura articular; aumento ou diminuição da sudorese na extremidade acometida; desmineralização óssea; perda muscular e diminuição da força na extremidade acometida.

Na nossa casuística observamos predomínio da fase II.

O diagnóstico de distrofia simpático reflexa é clínico e o estudo radiológico evidenciou osteopenia acentuada em 2 casos de nossa série.

O objetivo do tratamento é o alívio sintomático da dor e o aumento da capacidade funcional do membro acometido. Consideramos conveniente iniciar o tratamento com medidas não invasivas. Todos os pacientes submeteram-se a medidas de medicina física que incluíam a hidrotermoterapia e a cinesioterapia precoces.

Dos seis pacientes que obtiveram bons resultados, 3 faziam uso de medicação analgésica de ação central. A isto atribuímos o fatos de os medicamentos diminuírem a dor neuropática e conseqüentemente permitirem a mobilização e a recuperação funcional.

Pudemos constatar com este trabalho que o método preconizado para o tratamento de DSR é efetivo, com uma melhora significativa.

Verificamos que os pacientes que obtiveram os piores resultados foram aqueles que demoraram mais tempo para chegar ao nosso serviço, ou seja, aqueles que iniciaram o tratamento após um maior tempo de evolução da doença. Também observamos, assim como Eulry et al. (1990)(1) que os melhores resultados foram obtidos após um tempo maior de acompanhamento. Com isto pudemos estabelecer uma relação de melhores resultados em relação a precocidade do tratamento e maior tempo de acompanhamento.

Como todo equipamento do qual fizemos uso, é disponível na maioria dos centros de Medicina Física e Reabilitação, o seu manuseio não requer gastos adicionais e o custo operacional é baixo.

\section{Conclusão}

Os autores concluem que a abordagem reabilitacional precoce e o uso de medicação analgésica de ação central, estão correlacionados, nos casos estudados, com os bons resultados no tratamento da DSR no pé e no tornozelo.

Constituem vantagem do tratamento proposto, a simplicidade de execução, o baixo custo operacional e o método não invasivo.

\section{Referências Bibliográficas}

1. EURY, F.; ACZEL, F.; VASSEUR, P.; PATTIN, S.; VICENS, J. L.; FLAGEAT, J.; GAILARD, F.; DOURY, P. L'Algodystrophie du Pied. A propos de 199 observation. Revue de Rhumatisme. Vol 57(4), 351356, 1990.

2. PAYNE, R. Neuropathic Pain Syndromes, with Special Reference to Causalgia and Reflex Sympathetic Dystrophy. The Clinical Journal of Pain. Vol 2, 59-73, 1986.

3. International Association for the Study of Pain. Classification of chronic pain-descriptions of chronic pain syndromes and definitions of pain terms. Pain. Supplement 3, S29-30, 1986 (reprinted in 1991). 